\title{
Urban street mapping using Quickbird and Ikonos images
}

\author{
Renaud Péteri and Thierry Ranchin \\ Remote Sensing \& Modeling Group \\ Ecole des Mines de Paris \\ 06904 Sophia Antipolis, France \\ Email: renaud.peteri@ensmp.fr
}

\begin{abstract}
This article addresses the problem of urban street mapping from new high resolution satellite images. The proposed algorithm is divided in two sequential modules: a topologically correct graph of the street network is first extracted, and streets are then extracted as surface elements. The graph of the network is extracted by a following algorithm which minimizes a cost function. The surface extraction algorithm makes use of specific active contours (snakes) combined with a multiresolution analysis (MRA) for minimizing the problem of noise. This reconstruction phase is composed of two steps: the extraction of street segments and the extraction of street intersections. Results of the street network extraction are presented on Quickbird and Ikonos images. Future prospects are also exposed.
\end{abstract}

\section{URBAN STREET NETWORK EXTRACTION}

Among possibilities of remote sensing for urban uses, extraction of street networks is an important issue. An upto-date knowledge of the street network is necessary for urban planning, map updating or estimation of atmospheric or noise pollution. Moreover, an assisted street extraction from remotely sensed data can highly help cities which do not have a cartography of their street network. While the developed extraction method systems yield to very encouraging results when applied on rather simple scenes (i.e. rural areas), the automatic extraction of objects in complex scenes, such as dense urban areas, has turned out to be very challenging. Few methods focus on urban areas (one can mention [1] or [2]).

The recent possibility to have satellite images with a high spatial resolution (1 meter or less) has re-boosted the interest for street extraction in urban areas. This increased resolution enables a more accurate localization of the street sides as well as its extraction as surface element. In return, it generates a higher complexity of the image and an increase of geometric noise (vehicles, trees along the street, occlusions, ... ).

\section{THE PROPOSED APPROACH}

\section{A. Description}

A method has been developed in order to extract and characterize the street network from new high resolution images of urban areas. Inputs of the algorithm, besides the high resolution satellite image, are models of streets (using streets properties defined by [2]) and properties of street network (such as connexity). Our algorithm is decomposed in two sequential modules (fig.1). Firstly, a topologically correct graph of the street network is extracted. This step aims at giving correct spatial connections between streets as well as an approximation of their location. The next step is the actual street reconstruction. Due to the high resolution of the images, a surface reconstruction has to be performed. This step uses the previous step of graph management as an initialization.

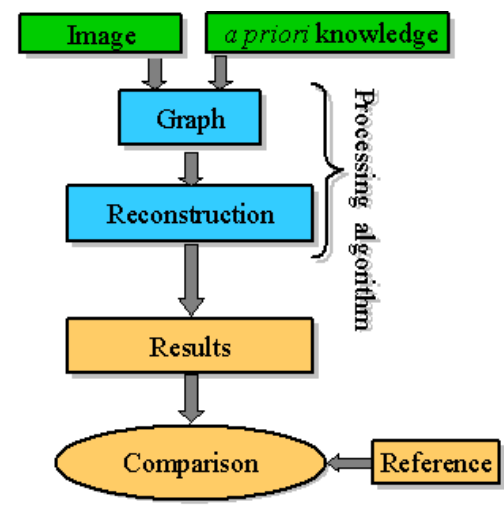

Fig. 1. The methodology including topology management and street reconstruction

In the next sections, the different modules of the process are more precisely described.

\section{B. Graph management}

This module intends to extract a topologically correct graph of the street network. It aims at giving correctly spatial connections between streets as well as an approximation of their location. The graph can come from a road database or be extracted automatically. In this second case, the graph extraction algorithm is the one of [3]: a following algorithm selects the best path for the potential road by minimizing a cost function. The cost function evaluates the homogeneity of the local radiometry variance for several propagation directions. At this step, the extracted graph is topologically correct but the different polylines are not necessary well registered.

From the extracted graph, polylines are then sampled and propagated along their normal direction in order to initialize the surface reconstruction module. 


\section{Surface reconstruction module}

1) Description: The aim of this module is to reconstruct streets as surface elements from the graph provided by the previous step. This module makes use of specific active contours (snakes) combined with a multiresolution analysis (MRA). The snake implementation is based on the greedy algorithm described by [4]. The use of the MRA with the Wavelet Transform enables to perform a multiresolution edge detection (see [5]). It also increases the algorithm convergence by minimizing the problem of noise (vehicles, ground markings,...). Two sequential steps compose this reconstruction phase: the extraction of street segments with parallel sides and the extraction of street intersections. Indeed, these two objects present too many differences in both topology and shape to be processed in the same way. The frontier between the two kinds of process is defined by a circle including the whole intersection (fig. 2).

2) Extraction of parallel street sides: For extracting portions of street with parallel sides, a new object has been defined: the DoubleSnake. It is composed of two branches which are two snakes evolving jointly. The DoubleSnake energy functional has a new term $E_{/ /}$that constrains the DoubleSnake to maintain a local parallelism between its two branches. Moreover, their extremity points are forced to minimize their energy staying on the intersection circle.

The snake energy functional is:

$$
E=\sum_{i}\left[\alpha^{i} E_{\text {cont }}^{i}+\beta^{i} E_{\text {curv }}^{i}+\gamma^{i} E_{2^{j}{ }_{\text {image }}}^{i}+\delta^{\mathbf{i}} \mathbf{E}_{/ /}^{\mathbf{i}}\right]
$$

where $i$ represents the point $i$ of one of the branch.

$E_{\text {cont }}^{i}$ and $E_{\text {curv }}^{i}$ are internal energies that control the snake's shape. A special attention is paid on the image energy term $E_{2^{j}{ }_{\text {image }}}$, as it the one that attracts the snake to the object of interest. The image energy is computed with the wavelet coefficients at different spatial resolutions:

$$
E_{2^{j}{ }_{\text {image }}^{i}}^{i}=-\sqrt{\left|W_{2^{j}}^{1} f(i)\right|^{2}+\left|W_{2^{j}}^{2} f(i)\right|^{2}}
$$

where $W_{2^{j}}^{1,2} f(i)$ are the coordinates of the wavelet transform and $j$ is the resolution in a dyadic analysis. A more detailed description of the energy terms can be found in [6].

3) Extraction of street intersections: Once street segment extraction is finished, the intersection extraction starts. They are extracted by simple snakes which are initialized by pairing extremity points of the DoubleSnakes (see fig. 2). This IntersectionSnake has its extremities fixed and is constrained not to go out of the circle.

4) Reconstruction algorithm flowchart: Fig. 3 represents the different steps of the reconstruction algorithm. From the original image, a multiresolution analysis is performed, giving several approximation images at coarser resolutions and several wavelet coefficient images. DoubleSnakes are first applied on the coarsest resolution image, then on each intermediate resolution images until they run on the original resolution image. Coefficients of the different energy terms are adapted to the image resolution. Once DoubleSnakes have all minimised their energy, the IntersectionSnakes are initialized and the extraction starts in the same multiresolution process.

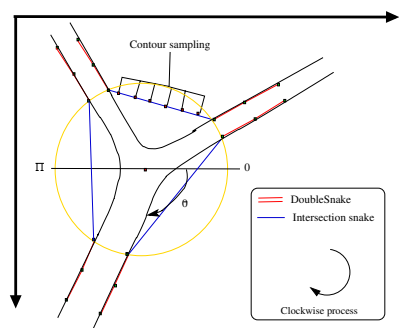

Fig. 2. Initialization of the intersection snakes

The algorithm stops when all snakes are in an equilibrium state.

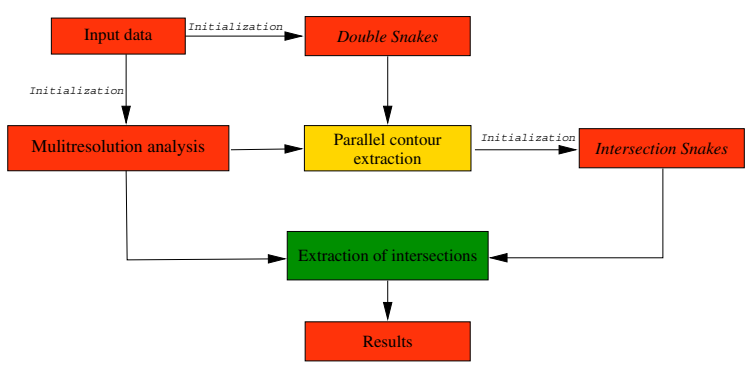

Fig. 3. Reconstruction algorithm

\section{RESULTS AND PROSPECTS}

\section{A. Real-case study}

As the graph module is currently under integration, only the reconstruction module will be illustrated in the following examples. Images of Fig. 4 and Fig. 5 come respectively from the Quickbird satellite (spatial resolution of 0.61 meter in the panchromatic mode) and the IKONOS satellite (spatial resolution of 1 meter). The input graph (coming from a database or manually given) has been superposed (4(a) and 5(a)). The first image 4(a) represents a straight street with high geometric noise (shadows and ground marking). Image 4(b) shows the extraction result after propagation, sampling of the input graph and the DoubleSnake evolution: the multiresolution approach has prevented the snake from being trapped by the noise. The second test image 5(a) includes a crossroad composed of two main streets. Image 5(b) shows the extracted street after propagation and sampling of the input graph, extraction of the parallel street sides and extraction of intersections. The circle delimiting the two kinds of the reconstruction process is also drawn. Contours have globally been extracted with a good precision (except the sharp edge of the intersection). For a more detailled illustration of the different steps the algorithm, see [7].

\section{B. Conclusion and future prospects}

This article describes a new method for extracting the street network from high resolution satellite images. A topologically 


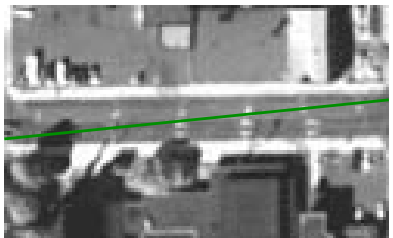

(a) Original image with the street network graph

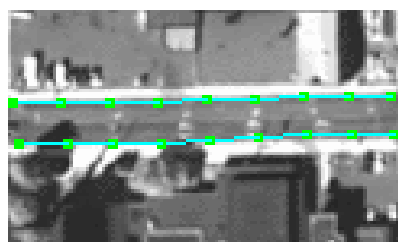

(b) Final result

Fig. 4. Test on a Quickbird image $(0.61 \mathrm{~m}$ spatial resolution $)$

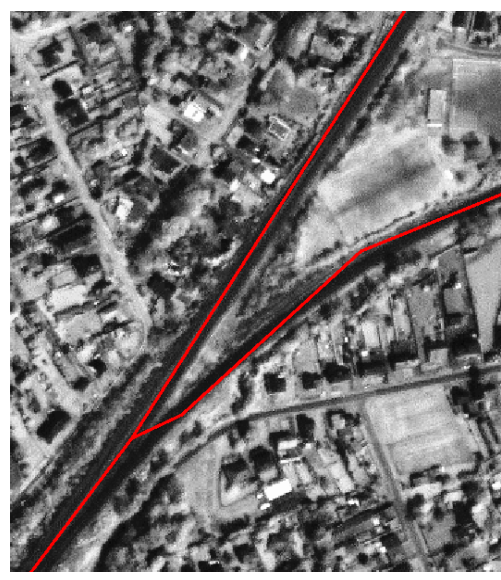

(a) Original image with the street network graph

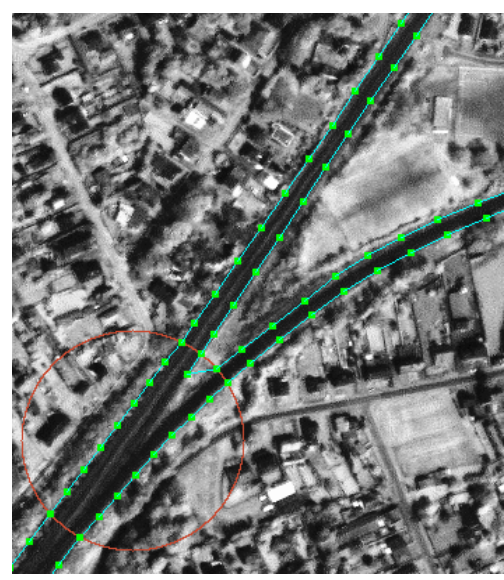

(b) Final result

Fig. 5. Test on an IKONOS image ( $1 \mathrm{~m}$ spatial resolution)

correct graph of the street network is first extracted, and streets are then extracted as surface elements. The graph of the network is extracted by a following algorithm [3] which minimizes a cost function. The surface extraction algorithm makes use of specific snakes combined with a multiresolution analysis for minimizing noise. Current works aim at increasing the robustness of the algorithm by taking the texture into account. The use the wavelet coefficients deriving from the MRA for extracting texture informations proper to streets could constraint the snake evolution. Some contextual information (such as building alignment) can also be a clue for the extraction. Results on test images are encouraging. However, results shoud be validated and characterized using quantitative criteria described in [8].

\section{ACKNOWLEDGMENT}

This work is supported by a CNRS/DGA grant of the french Ministry of Defence. Thanks go to Sylvain Airault from the French National Geographic Institute for having provided the source of his program. The authors would also like to thank the firm G.I.M. (Geographical Information Mapping) for the IKONOS image of the Hasselt area and the PNTS project for the Quickbird image of Strasbourg.

\section{REFERENCES}

[1] S. Hinz, A. Baumgartner, and E. Heinrich, "Modeling contextual knowledge for controlling road extraction in urban areas," in Proceedings of the IEEE/ISPRS Joint Workshop on Remote Sensing and Data Fusion over Urban Areas, Rome, Italy, November 2001.

[2] I. Couloigner and T. Ranchin, "Mapping of urban areas : A multiresolution modeling approach for semi-automatic extraction of streets," Photogrammetric Engineering and Remote Sensing, vol. 66, no. 7, pp. 867-874, 2000.

[3] S. Airault and O. Jamet, "Détection et restitution automatique du réseau routier sur des images aériennes," Traitement du Signal, vol. 12, no. 2, pp. 189-200, 1995.

[4] D. Williams and M. Shah, "A fast algorithm for active contours and curvature estimation," in CVIP : Image Understanding, vol. 55, January 1992, pp. 14-26.

[5] S. Mallat, A Wavelet Tour of Signal Processing. London: AP Professional, 1997.

[6] R. Péteri and T. Ranchin, "Multiresolution snakes for urban road extraction from ikonos and quickbird images," in 23nd EARSeL Annual Symposium "Remote Sensing in Transition", Ghent, Belgium, 2-5 June 2003, to appear.

[7] R. Péteri, J. Celle, and T. Ranchin, "Detection and extraction of road networks from high resolution satellite images," in Proc. IEEE International Conference on Image Processing, Barcelona, Spain, September 2003, to appear.

[8] R. Péteri, I. Couloigner, and T. Ranchin, "How to assess quantitatively road extracted from high resolution imagery?" Photogrammetric Engineering \& Remote Sensing, 2003, to appear. 